\title{
Thrombus burden quantification: an evolving tool for coronary interventions
}

\section{Introduction}

Thrombus is a distinctive histo-pathological feature of patients presenting with STelevation myocardial infarction (STEMI) and constitutes an adverse finding that significantly affects interventional management. Characterization and quantification of coronary thrombi have been a challenge since the beginning of thrombolysis era. Almost 20 years ago, the Thrombolysis in Myocardial Infarction (TIMI) group developed an angiographic thrombus scoring system, relating the maximum thrombus length to the reference vessel diameter. These investigators described 6 thrombus grades (G0 to G5), assigning grade $\mathrm{G} 5$ to complete total occlusions [1]. Once the safety and effectiveness of coronary stenting in thrombus containing lesions was proven [2], several investigations consistently demonstrated an association between thrombus burden (TB) and tissue perfusion parameters. However, most of these early studies lacked statistical power to evaluate late clinical outcomes [3].

A crucial turning point in the angiographic evaluation of thrombus was the findings of the Rotterdam group [4,5]. Their main contribution was the exclusion of cases with persistent complete occlusion in which it was not possible to evaluate the amount of thrombus. In addition, these patients tended to be associated with a poor prognosis due to failed reperfusion. The remaining patients were classified into large (thrombus length $\geq 2$ reference vessel diameter) and small TB categories. Notably, a significantly higher rate of the combined clinical outcome measure (death, repeat myocardial infarction, or infarct-related artery revascularization) was found in the large TB group $(24.9 \%$ vs $15.3 \%$, $\mathrm{p}<0.001)$. Interestingly, this excess of events was closely related to the high rates of stent thrombosis $(8.2 \%$ vs $1.3 \%, \mathrm{p}<0.001)$, a finding attributable to the almost exclusive use of first generation drug-eluting stents (DES) and to the 3-6 months duration of dual antiplatelet treatment, according to initial recommendations.

To validate these findings, we carried out a prospective study including 504 consecutive STEMI patients systematically treated with primary percutaneous coronary intervention (PCI), using mostly bare-metal stents and second generation DES and prolonging the second antiplatelet drug for at least 12 months [6]. Roughly half of the patients (47\%) fulfilled large TB criteria (length $\geq 2$ vessel diameter or large thrombus during aspiration). Interestingly, this group received more frequently adjuvant medical or mechanical therapies $(62 \%$ abciximab, $34 \%$ thrombus aspiration). Despite doubling the incidence of acute angiographic complications, such as distal embolization or no-reflow phenomenon, the group with large TB eventually was not associated with an increased incidence of late clinical events (main clinical outcome measure $12.8 \%$ vs $16.2 \%, \mathrm{p}=0.691$ ) or stent thrombosis $(2.2 \%$ vs $0.5 \%, \mathrm{p}=0.190)$ after a mean follow-up of 19 months.

Given the available evidence, it seems that the clinical impact of TB depends on the rate of stent thrombosis, and therefore on the type of device used and the intensity and duration of antiplatelet therapy. Accordingly, a systematic and comprehensive analysis of TB may take sense in the era of bioresorbable vascular scaffolds, as STEMI patients appear to represent an attractive target for the use of these novel devices [7]. Also, TB assessment may help to identify patients for alternative strategies such as "delayed stenting", given the conflicting results of its routine application [8].

On the other hand, we should keep in mind that angiography is a limited technique in
David Martí Sánchez ${ }^{1 *}$ and Fernando Alfonso ${ }^{2}$ 'Department of Cardiology, Hospital Central de la Defensa, Spain

2Department of Cardiology, Hospital Universitario de La Princesa, Spain

*Author for correspondence: docalcala@hotmail.com Tel: +34914222363

Submitted: 13 March 2017

Accepted: 28 March 2017

Published online: 03 April 2017 
the diagnosis of thrombus, and its sensitivity is not greater than $50-60 \%$ in detecting small or moderate sized thrombus [9]. In addition, angiographic interpretation of thrombus is highly subjective, mainly due to the difficulty of characterizing elements such as reduced contrast density or irregular lesion contour, and to discriminate the interface between thrombus and atheroma plaque. In this regard, in our study we found significant interobserver discrepancies in almost $30 \%$ of the cases [6]. Fortunately, advances in intracoronary imaging, and specifically the development of optical coherence tomography (OCT), have overcome most of the limitations of angiography in the detection, characterization, and quantification of coronary thrombus (Figures 1 and 2).
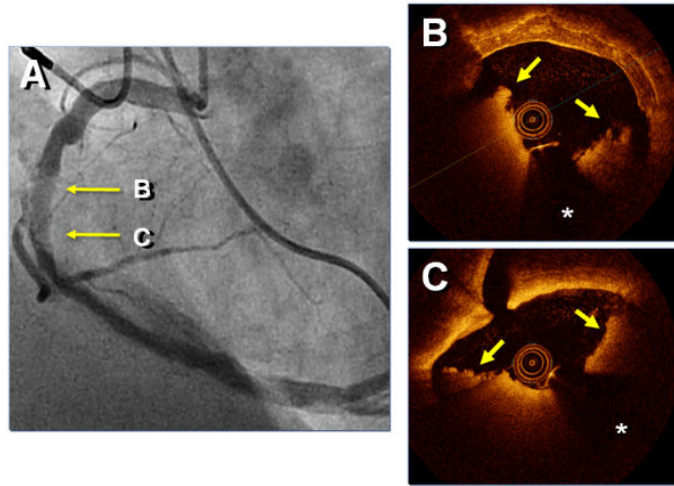

Figure 1: Patient presenting late and asymptomatic after an inferior STEMI. A) coronary angiography showing a large thrombus burden in the mid segment of the right coronary artery. A large, but unclear, angiographic filling defect causing major haziness is demonstrated (arrows). B and C) Optical coherence tomography findings disclose a large red thrombus (arrows) casting a large dorsal shadow and severely reducing the vessel lumen. $\left(^{*}\right)$ Denotes wire artefact.

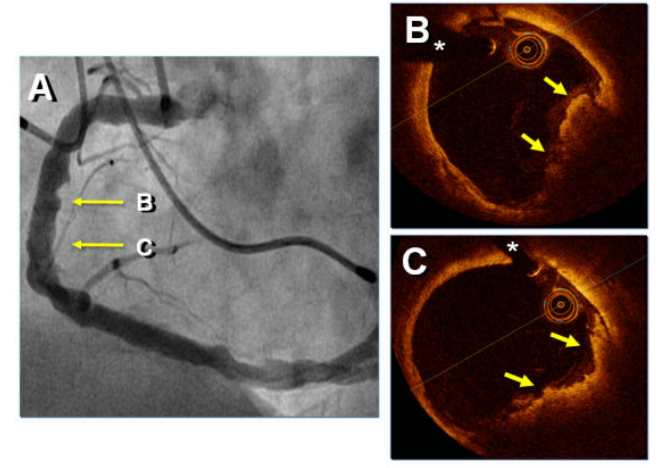

Figure 2: The same patient after intracoronary abciximab and 1 week of dual antiplatelet therapy. A) Thrombus resolution was demonstrated on coronary angiography (arrows). B and C) Optical coherence tomography unravelled persistence of small residual thrombus but a drastic reduction in thrombus burden. $\left({ }^{*}\right)$ Denotes wire artefact.
Notably, some OCT based thrombus scores have already been developed. Prati et al. designed a simple quantification system by summing the absolute number of quadrants occupied by thrombus through all of the pullback frames. This methodology allowed the documentation of larger thrombus resolution with local abciximab delivery through a dedicated catheter compared with the administration of this drug through the guiding catheter [10]. More recently, in a sub analysis of the Trial of Routine Aspiration Thrombectomy with PCI versus PCI Alone in Patients with STEMI (TOTAL) trial, Kajander et al. observed that TB characterization by OCT during primary PCI was feasible in the vast majority $(86 \%)$ of patients. In contrast to other studies, these researchers defined thrombus area (lumen area minus flow area), TB (mean thrombus area divided by mean lumen area) and thrombus volume (mean thrombus area at each interval multiplied by the length of the stent). Importantly, they found these measures as highly reproducible among different observers [11]. The main limitation of OCT to measure $\mathrm{TB}$ is that red thrombus cast major shadow obscuring its dorsal border. In any case, accumulating clinical experience with OCT is providing novel diagnostic insights and allows a more precise characterization of intracoronary thrombus [12].

\section{Conclusion}

In conclusion, $\mathrm{TB}$ seems to be associated to a higher device-related incidence of stent thrombosis. Evaluation of $\mathrm{TB}$ with modern imaging techniques may tailor the need of adjuvant therapies, as well as guide device selection and optimization. However, further definition of standardized OCT optimization criteria and also optimal surrogate and clinical endpoints will be required to improve acute and longterm results of coronary interventions in this challenging anatomic setting.

\section{References}

1. Gibson CM, de Lemos JA, Murphy SA, et al. Combination therapy with abciximab reduces angiographically evident thrombus in acute myocardial infarction: a TIMI 14 substudy. Circulation. 103: 2550-2554 (2001).

2. Alfonso F, Rodriguez P, Phillips P, et al. Clinical and angiographic implications of coronary stenting in thrombuscontaining lesions. J. Am. Coll. Cardiol. 29(4): 725-733 (1997).

3. Topaz O. Revascularization of thrombus-laden lesions in AMI - the burden on the interventionalist. J. Invasive. Cardiol. 19(8): 324-325 (2007).

4. Sianos G, Papafaklis MI, Daemen J, et al. Angiographic stent thrombosis after routine use of drug-eluting stents in STsegment elevation myocardial infarction: the importance of thrombus burden. J. Am. Coll. Cardiol. 50(7): 573-583 (2007).

5. Sianos G, Papafaklis MI, Serruys PW. Angiographic thrombus burden classification in patients with ST-segment elevation myocardial infarction treated with percutaneous coronary intervention. J. Invasive. Cardiol. 22: 6B-14B (2010). 
6. Martí D, Salido L, Mestre JL, et al. Impact of thrombus burden on procedural and mid-term outcomes after primary percutaneous coronary intervention. Coron. Artery. Dis. 27(3): 169-175 (2016).

7. Alfonso F, Cuesta J, Bastante T, et al. Bioresorbable vascular scaffolds in patients with acute myocardial infarction: a new step forward to optimized reperfusion? J. Thorac. Dis. 8(6): E417-423 (2016).

8. Kelbaek H, Hofsten DE, Kober L, et al. Deferred versus conventional stent implantation in patients with ST-segment elevation myocardial infarction (DANAMI 3-DEFER): an open-label, randomised controlled trial. Lancet. 387(10034): 2199-2206 (2016).

9. den Heijer P, Foley DP, Escaned J, et al. Angioscopic versus angiographic detection of intimal dissection and intracoronary thrombus. J. Am. Coll. Cardiol. 24(3): 649-654 (1994).

10. Prati F, Di Vito L, Ramazzotti V, et al. Randomized trial of standard versus Clear Way-infused abciximab and thrombectomy in myocardial infarction: rationale and design of the COCTAIL II study. J. Cardiovasc. Med. 14(5): 364371 (2013).
11. Kajander OA, Koistinen LS, EskolaM, et al. Feasibility and repeatability of optical coherence tomography measurements of pre-stent thrombus burden in patients with STEMI treated with primary PCI. Eur. Heart. J. Cardiovasc. Imaging. 16(1): 96-107 (2015)

12. Alfonso F, Cuesta J, Bastante T, Rivero F, Jiménez S. Calcified nodule mimicking red thrombus on optical coherence tomography. JACC. Cardiovasc. Interv. 8(1): 120-121 (2015). 